\title{
A new membrane re-anchored protein originating from GPC3 against hepatoma cells HepG2
}

\author{
DONGYE YANG, JUNWEN YANG, FANGGEN LU, CAIHONG LI, JINGBO YANG and JIELING LIANG \\ Department of Digestive Diseases, the Second XiangYa Hospital of Central South University, \\ Furong, Changsha, Hunan 410011, P.R. China
}

Received April 1, 2011; Accepted July 13, 2011

DOI: $10.3892 / \mathrm{mmr} .2011 .536$

\begin{abstract}
The aim of this study was to confirm the localization of recombinant pGPC3+afp-EGFP which expressed a new re-anchored protein named GPC3+afp-EGFP on the cytoplasmic membrane and to investigate its functions against hepatocellular carcinoma (HCC). EGFP expression in transfected HepG2 cells was observed using fluorescence and a confocal microscope. pGPC $3+$ afp-EGFP expression was detected in membranous and soluble proteins extracted from transfected human embryonic kidney 293 cells by Western blot analysis using GPC3 mAb. The proliferation of transfected HepG2 cells with pGPC3+afp-EGFP (experimental group) was detected using SRB assay and compared to those of transfected HepG2 cells with pGPC3 (control group) and non-transfected HepG2 cells (blank group). Quantitative analysis of mRNA expression of the Fas gene was conducted by real-time PCR using the $\beta$-actin housekeeping gene as the internal control at variable times. Apoptotic HepG2 cells in the three groups were counted and statistically analyzed by a contingency table Chi-square test using Spss 11.5 software and TUNEL assay. Production of both TNF- $\alpha$ and IFN- $\gamma /$ IL2 was detected by ELISPOT after co-cultivation of transfected HepG2 cells with peripheral blood lymphocytes at different time-points in the experimental group. Green fluorescence was mainly found around the transfected HepG2 cell periphery through fluorescence and confocal microscopy. GPC3+afp-EGFP could not be detected in soluble protein but only in membranous protein. Proliferation curves showed that the proliferative quantities of transfected HepG2 cells in the experimental group decreased, whereas the mRNA expression of the Fas gene increased significantly compared to those of the other two groups. The numbers of apoptotic cells in the experi-
\end{abstract}

Correspondence to: Dr Dongye Yang, Department of Digestive Diseases, the Second XiangYa Hospital of Central South University, 139 Renmin Middle Road, Furong, Changsha, Hunan 410011, P.R. China

E-mail: dongyeyang@yahoo.com.cn

Key words: strong epitope $\mathrm{hAFP}_{542-550}$, glypican 3, protein engineering, localization, anti-HepG2 mental group were significantly higher compared to those in the other two groups, as shown by statistical analysis. Both TNF- $\alpha$ and IFN- $\gamma /$ IL2 were induced and were much higher in the experimental groups than in the diverse control groups at variable times. A new re-anchored protein GPC3+afp-EGFP expressed by recombinant pGPC $3+$ afp-EGFP was localized on the cytoplasmic membrane, and had multiple functions against HCC, such as inhibition of transfected HepG2 cell proliferation, promotion of transfected HepG2 apoptosis and induction of antitumor cytokine excretion.

\section{Introduction}

$\alpha$-Fetoprotein (AFP) is often expressed in human hepatocellular carcinoma (HCC), and its measurement in serum plays an important role in diagnosing and monitoring responses to treatment (1). The idea that AFP can serve as a target for immunotherapy is not novel: peptide fragments of AFP presented in the context of major histocompatibility molecules could serve as potential recognition targets by CD8 T cells, such as hAFP ${ }_{542-550}$ (GVALQTMKQ), a 9-mer peptide derived from the AFP protein (2). Furthermore, hAFP 542-550 $_{\text {peptide- }}$ generated $\mathrm{T}$ cells from both human lymphocyte cultures and A2.1/Kb transgenic mice recognized AFP-transfected targets in both cytotoxicity assays and cytokine release assays (3). These findings clearly establish AFP as a potential target for T cell-based immunotherapy. Glypican-3 (Gpc3), a cell surface-linked heparan sulfate proteoglycan, is a glycosylphosphatidylinositol anchored protein and has been considered to be a novel serum marker for HCC (4). Based on the 'protein engineering' theory a recombinant of pGPC3+afp-EGFP was constructed successfully, and its expression and localization were demonstrated in our previous study (5). In that study, we further confirmed re-anchored protein GPC3+afp-EGFP localization on the cytoplasmic membrane and investigated its functions against HCC.

\section{Materials and methods}

Sublocalization of GPC3+afp-EGFP in cells. HepG2 hepatoma cells $\left(\mathrm{GPC}^{+} \mathrm{AFP}^{+}\right)$and human embryonic kidney (HEK) 293 cells $\left(\mathrm{GPC}^{-} \mathrm{AFP}^{-}\right)$were cultured in DMEM (Gibco, Invitrogen Corp., Carlsbad, CA, USA) supplemented with $10 \%$ fetal bovine serum (FBS) (Hyclone, Logan, UT, USA) 
containing $1 \%$ penicillin and streptomycin, and maintained in a humidified incubator under $5 \% \mathrm{CO}_{2}$ at $37^{\circ} \mathrm{C}$. Cells were seeded into 6-well plates or flasks and cultured overnight to allow a cell confluence of 70-80\%. pGPC3+afp-EGFP was transfected into cells via lipofectamine 2000 (Invitrogen) in Opti-MEM medium (Invitrogen) according to the manufacturer's instructions.

Fluorescence microscopy. pGPC3+afp-EGFP was transfected into HepG2 cells via lipofectamine 2000 using pEGFP-N1 transfection as the positive control. EGFP expression in both groups was observed using a fluorescence microscope (NIKON TE2000-U) 24 and $48 \mathrm{~h}$ after transfection.

Confocal microscopy. Transfected HepG2 cells with pGPC3+afp-EGFP were placed in a $37^{\circ} \mathrm{C}$ heated chamber (Zeiss S-Type incubator) and imaged on a Zeiss LSM 510 confocal microscope with a $\mathrm{x} 40$ oil objective (NA 1.3) at radial and axial resolutions of 0.4 and $1.0 \mu \mathrm{m}$, respectively. For ratiometric imaging, cells were excited at $488 \mathrm{~nm}$, emission was detected at 505-550 nm, and a DIC transmission image was acquired simultaneously.

Fractional proteins detection. After membranous proteins and soluble proteins were extracted from transfected HEK 293 cells by using the ProteoExtract Native Membrane Protein Extraction kit (Merck KGaA, Darmstadt, Germany), GPC3+afp-EGFP expression was detected by Western blotting in which mouse anti-human GPC3 monoclonal antibody (cat num: AF2119; Calbiochem, San Diego, CA, USA) was used as the primary antibody, and HRP anti-mouse $\operatorname{IgG}$ as the secondary antibody (1:5000).

Functions study against HepG2 hepatoma cells. Peripheral blood lymphocytes (PBLs) were isolated from fresh peripheral blood of healthy human volunteers by Lymphoprep ${ }^{\mathrm{TM}}$ lymphocyte separation medium (Axis Shield Poc AS, Oslo, Norway). PBLs were cultured in RPMI-1640 (Gibco BRL, Invitrogen Corp.,) supplemented with 10\% FBS (HyClone, Logan, UT, USA) containing $1 \%$ penicillin and streptomycin (Gibco, Invitrogen Corp.).

Lymphocyte cytotoxicity test by SRB assay. PBLs were adjusted to an appropriate concentration for the following step: HepG2 cells transfected with pGPC $3+$ afp-EGFP or pGPC 3 as the experimental or control group, respectively were co-cultured with PBLs in 96-well plates, and approximately 1,000 transfected HepG2 cells were seeded in each well mixed with 50,000 PBLs (1:50) using non-transfected HepG2 cells as the blank control group.

After $0,24,48$ and $72 \mathrm{~h}$ of co-cultivation, the cells from each group were collected and assayed using Sulforhodamine B (SRB, Sangon Biotech, Shanghai Co., Ltd., China) (6). OD values of $490 \mathrm{~nm}$ were mensurated and the proliferation curves were drawn according to the mean values.

Quantitative analysis of Fas gene mRNA expression by realtime PCR. RNAs were extracted from cells of the above three groups at $0,24,48$ and $72 \mathrm{~h}$ after co-cultivation with PBLs (3 wells for each sample). Reverse transcription reaction was carried out at $42^{\circ} \mathrm{C}$ for $60 \mathrm{~min}$, and $70^{\circ} \mathrm{C}$ for $5 \mathrm{~min}$. cDNA templates were diluted 10 times for the next step. For fluorescence quantitative PCR, the primers used were as follows: [Fas gene (150 bp) forward, tccaagggattggaattgag and reverse, acctggaggacagggcttat; $\beta$-actin gene (150 bp) forward, ctcttccagccttccttcct and reverse, agcactgtgttggegtacag]. The reaction system included the following components: SYBR (2X) $5 \mu 1$, forward and reverse primer $0.2 \mu 1$, respectively, Rox Reference Dye (50X) $0.2 \mu \mathrm{l}$, sterile water $3.4 \mu \mathrm{l}$ and cDNA template $1.0 \mu \mathrm{l}$. The qPCR mixture of Fas was amplified in the instrument of ABI PRISM 7900HT using the $\beta$-actin housekeeping gene as the control. The standard amplification curves of Fas and $\beta$-actin primers were drawn according to the threshold cycles (CT) from cDNA samples of 5-fold serial dilution, and the slopes of these curves would help to determine whether the two pairs of primers have similar amplification efficiency. Then the samples were amplified by these two pairs of primers, respectively and the $\mathrm{CT}$ values were mensurated.

Terminal deoxynucleotidyl transferase dUTP nick end labeling (TUNEL) assay. Approxixmately 5,000 transfected HepG2 cells from each group were added into each well and cultured for 4-6 h. They were adhered to sterilized cover slips which were placed on the bottom of 24-well plates, then PBLs were added in each well at the ratio of 50:1. At the end of $72 \mathrm{~h}$ after co-cultivation, cells were adhered to sterilized cover slips, and were fixed and stained according to the instructions of the Colorimetric TUNEL apoptosis assay kit (cat num: C1091; Beyotime Institute of Biotechnology, Shanghai, China). Apoptotic cells of each group were counted and statistically analyzed.

Enzyme-linked immunosorbent spot assay. After the transfected HepG2 cells with pGPC3+afp+EGFP were cultured for $24 \mathrm{~h}, \mathrm{pGPC} 3+\mathrm{afp}+\mathrm{EGFP} / \mathrm{HepG} 2$ cells were seeded into 96-well polyvinylidene fluoride-backed microplates of Human IFN $\gamma /$ IL-2 Dual Elispot and human TNF- $\alpha$ Elispot kits (U-Cytech bioscience Inc., Netherlands), respectively. PBLs were then added and incubated together for 14, 22, 28, 36 and $48 \mathrm{~h}$ at $37^{\circ} \mathrm{C}$.

Three kinds of groups were used as the controls, including a positive control of PBLs stimulated by $2.5 \mu \mathrm{g} / \mathrm{ml}$ phytohemagglutinin (PHA; Sigma, St. Louis, MO, USA), a negative control of blank HepG2 cells mixed with only PBLs, and a background control of RPMI-1640 with 10\% FBS.

The cells from the above groups were incubated for $22 \mathrm{~h}$ at $37^{\circ} \mathrm{C}$. At the end of incubation the cells were removed and the polyvinylidene fluoride-backed microplates were processed according to the manufacturer's instructions (4 wells in each group).

The number of spots was enumerated using an automated ELISPOT analyzer (BioReader 4000, Bio-Sys, Karben, Germany) designed to detect spots with predetermined criteria based on size, shape, and colorimetric density (Dakewe Biotech Company, Shenzhen, China).

\section{Results}

GPC3+afp-EGFP localization on the cytoplasmic membrane. After pGPC3+afp-EGFP and pEGFP-N1 were transfected into 
A

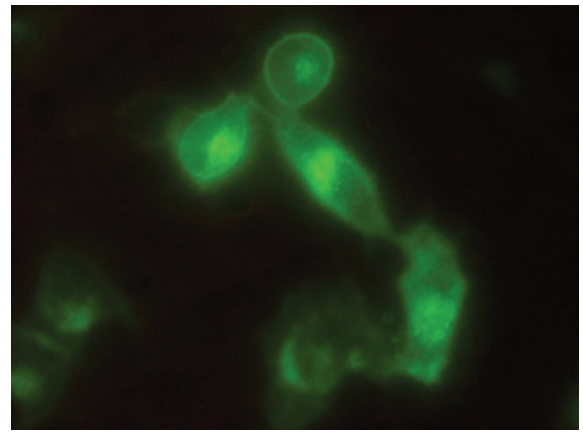

C

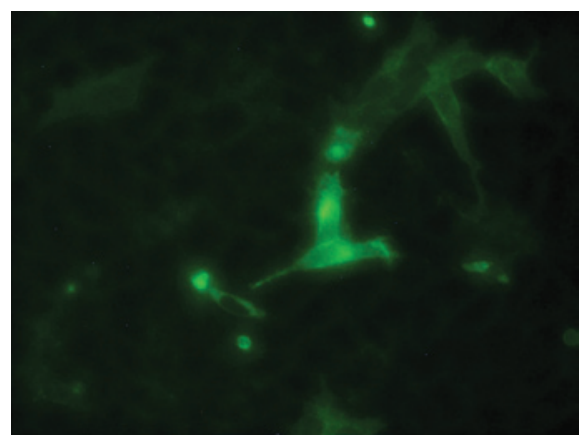

B

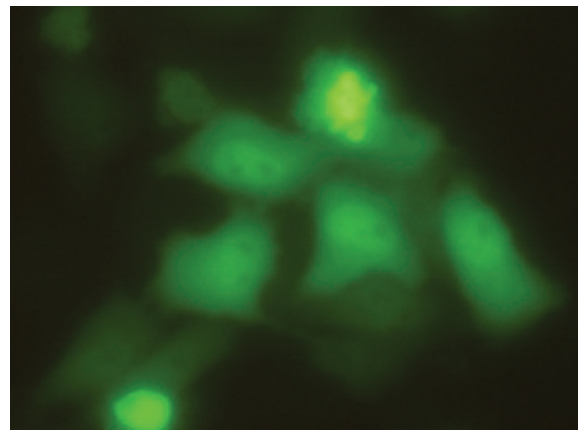

D

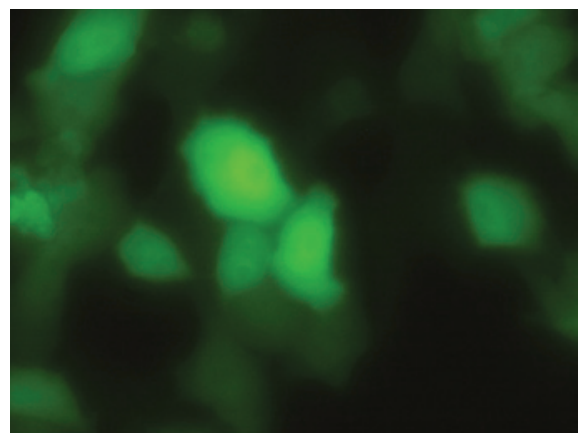

Figure 1. EGFP expression after pGPC3+afp-EGFP and pEGFP-N1 transfection into HepG2 cells (x40). (A) Lipo-pGPC3+afp-EGFP at $24 \mathrm{~h}$ post-transfection. (B) Lipo-pEGFP-N1 at $24 \mathrm{~h}$ post-transfection. (C) Lipo-pGPC3+afp-EGFP at $48 \mathrm{~h}$ post-transfection. (D) Lipo-pEGFP-N1 at $48 \mathrm{~h}$ post-transfection. Lipo, lipofectamine.

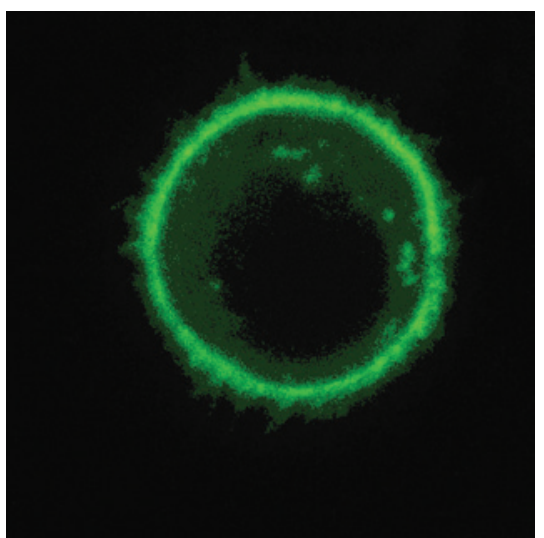

Figure 2. Expression of EGFP under a confocal microscope after pGPC3+afpEGFP transfection into HepG2 cells.

HepG2 cells, green fluorescence was mainly found around the periphery of pGPC3+afp-EGFP/HepG2 cells with sporadic distribution in the cytoplasm using a fluorescence microscope at 24 and $48 \mathrm{~h}$ after transfection, but the fluorescence of the pEGFP-N1/HepG2 cells was evenly distributed in the entire cell (Fig. 1). Green fluorescence was also observed around the periphery of pGPC3+afp-EGFP/HepG2 cells by confocal microscopy (Fig. 2).

Re-anchored protein GPC3+afp-EGFP (molecular weight was 93,700 daltons analyzed by DNAstar software) was confirmed to be expressed only in membranous proteins, but not in soluble proteins of the pGPC $3+$ afp-EGFP transfected groups at 24 and $48 \mathrm{~h}$ after transfection. There was no specific expression in the membranous or soluble proteins of blank HepG2 cells groups (Fig. 3).

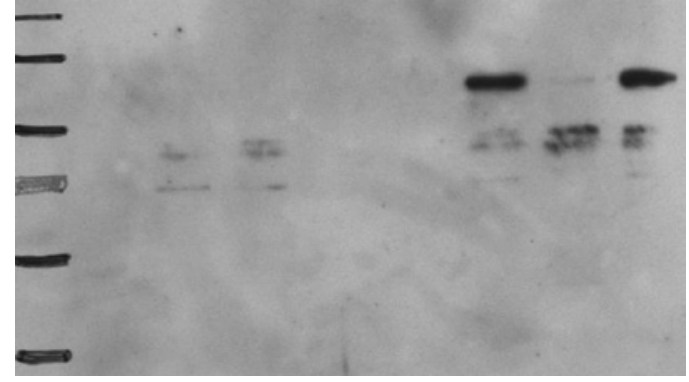

Figure 3. Expression of GPC3+afp-EGFP in soluble and membranous proteins by Western blotting in transfected HEK 293 cells. Lane 1, blank cell $\mathrm{SP}$ at $24 \mathrm{~h}$; lane 2, blank cell SP at $48 \mathrm{~h}$; lane 3, blank cell MP at $24 \mathrm{~h}$; lane 4 , blank cell MP at $48 \mathrm{~h}$; lane 5, lipo-pGPC3+afp-EGFP SP at $24 \mathrm{~h}$; lane 6, lipo-pGPC3+afp-EGFP MP at $24 \mathrm{~h}$; lane 7, lipo-pGPC3+afp-EGFP SP at $48 \mathrm{~h}$; lane 8, lipo-pGPC3+afp-EGFP MP at $48 \mathrm{~h}$. SP, soluble protein; MP, membranous protein; lipo, lipofectamine.

Lymphocyte cytotoxicity test by SRB and proliferation curves. $\mathrm{OD}$ value was measured at $490 \mathrm{~nm}$ by the SRB assay. The growth curves were drawn based on the average values of different samples. $F=1.615(p=0.136)$, which meant that the total variance was even. $\mathrm{F}=114.841(\mathrm{p}<0.01)$ among the different groups using univariate analysis of variance through Spss 11.5. software (shown in Table I and Fig. 4).

TUNEL assay. Apoptotic positive cells were stained yellow in the TUNEL assay. The positive cell numbers of the blank HepG2 group (blank control), the pGPC3 transfected HepG2 group (negative control), and the pGPC3+afp-EGFP transfected HepG2 group (experimental group) were 34, 61 and 132 in per 1,000 cells, respectively. The Pearson Chi-square value was $73.271(\mathrm{p}<0.01)$ analyzed by a contingency table 
Table I. OD values of $490 \mathrm{~nm}$ at $0,24,48$ and $72 \mathrm{~h}$, as shown by SRB assay.

\begin{tabular}{lcccc}
\hline Groups/time-point (h) & 0 & 24 & 48 & 72 \\
\hline Blank & 0.330 & 0.775 & 0.964 & 1.152 \\
(Blank HepG2) & 0.307 & 0.899 & 0.950 & 1.171 \\
& 0.319 & 0.898 & 1.088 & 1.227 \\
mean & 0.282 & 0.911 & 1.107 & 1.220 \\
Control & 0.310 & 0.871 & 1.027 & 1.193 \\
(pGPC3 transfected & 0.401 & 0.765 & 0.770 & 0.911 \\
HepG2) & 0.384 & 0.720 & 0.917 & 0.854 \\
& 0.321 & 0.693 & 0.815 & 1.309 \\
mean & 0.360 & 0.686 & 0.729 & 0.670 \\
Experimental & 0.337 & 0.728 & 0.668 & 0.487 \\
(pGPC3+afp-EGFP & 0.335 & 0.701 & 0.593 & 0.623 \\
transfected HepG2) & 0.328 & 0.681 & 0.678 & 0.379 \\
& 0.349 & 0.633 & 0.582 & 0.364 \\
mean & 0.337 & 0.686 & 0.630 & 0.463 \\
\hline
\end{tabular}

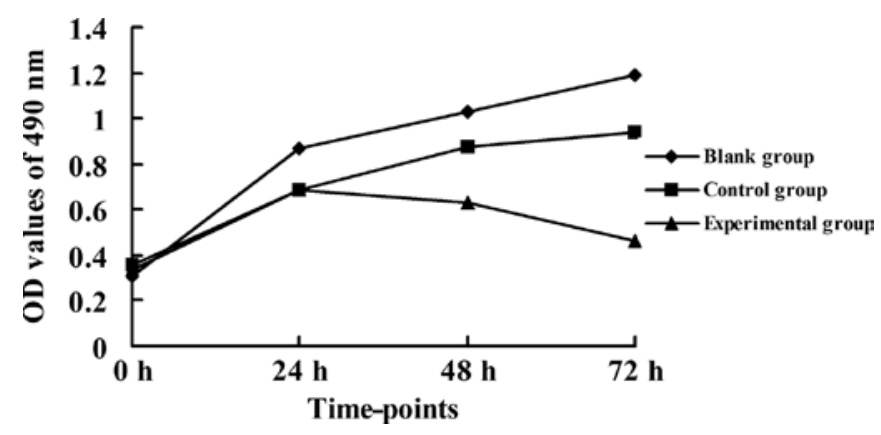

Figure 4. Proliferation curves of the three groups.

Chi-square test using Spss 11.5 software. The numbers of positive cells in the three groups were significantly different. Moreover, the experimental group had the highest incidence of apoptosis (Fig. 5).

Fluorescence quantitative PCR. The fact that the amplification curve slope of Fas was -3.078 and the correlation coefficient was 0.993 while those of $\beta$-actin were -3.072 and 0.994 , respectively demonstrated that these two pair primers had similar amplification efficiency. That the dissociation curves of both Fas and $\beta$-actin had a single peak indicated that the two pair primers were specific. $\mathrm{F}=1.876(\mathrm{p}=0.096)$ in homogeneity analysis of variance for $\Delta \mathrm{CT}$ values of each group, which meant that the total variance was even. $\mathrm{F}=794.926$ among the three groups $(\mathrm{p}<0.01)$. The above results show that Fas gene transcription in the experimental group was significantly higher than that in the other two groups using the Bonferroni method (Fig. 6, Tables II and III).
A

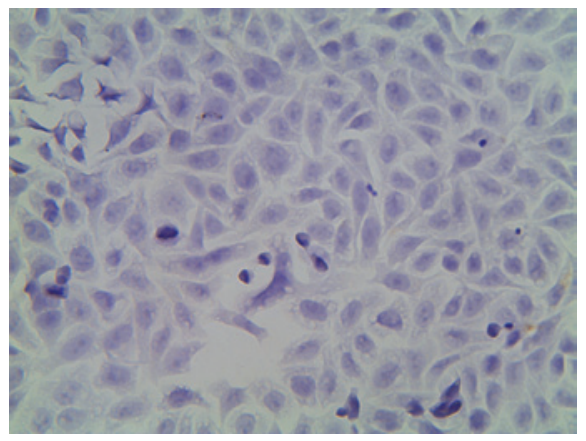

B

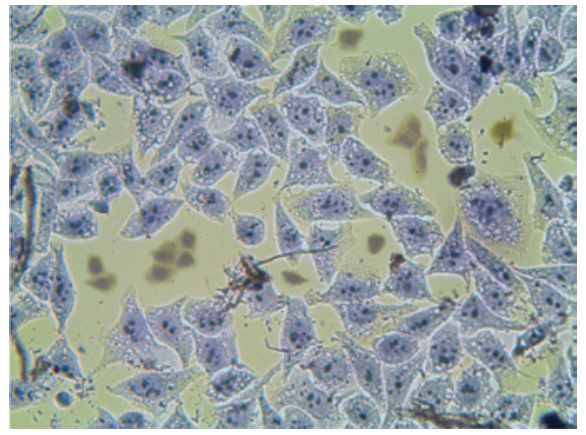

C

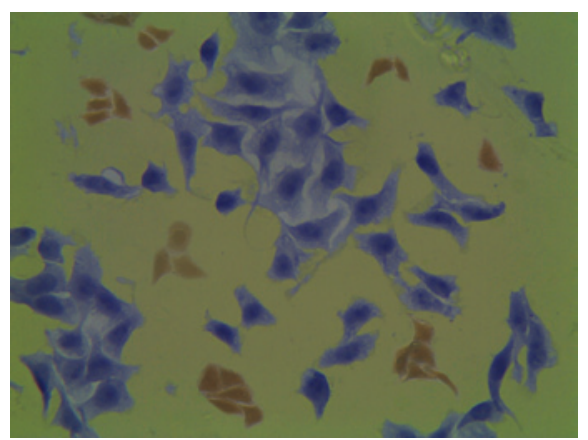

Figure 5. Typical images of apoptotic HepG2 cells in (A) the blank HepG2 group, (B) pGPC3 transfected HepG2 group and (C) pGPC3+afp-EGFP transfected HepG2 group at $72 \mathrm{~h}$ after co-cultivation with PBLs. The apoptotic cells were stained yellow $(\mathrm{x} 40)$.

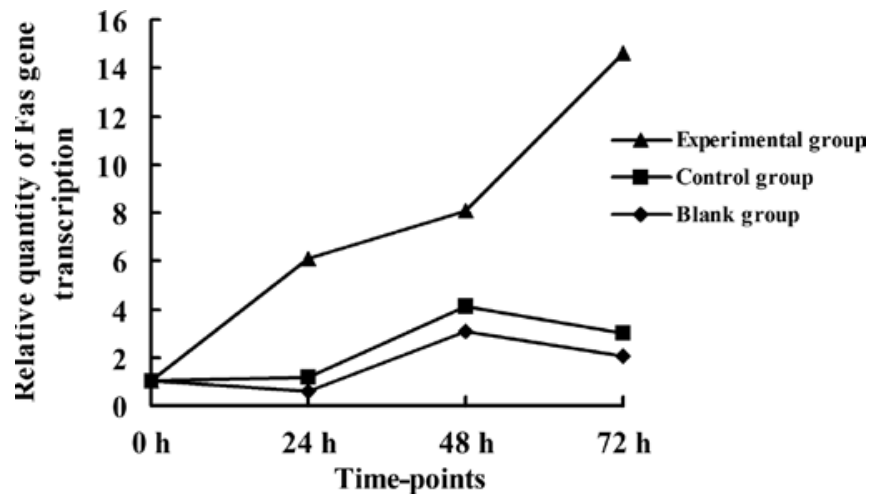

Figure 6. Tendency of the relative quantity of Fas gene transcription at different time-points.

Enzyme-linked immunosorbent spot assay. ELISPOT results were expressed by the numbers of spots. Compared to the negative and background controls, both TNF- $\alpha$ and IFN- $\gamma /$ IL2 were induced by the transfected HepG2 cells in all the experimental groups. The maximum production of TNF- $\alpha$ appeared 


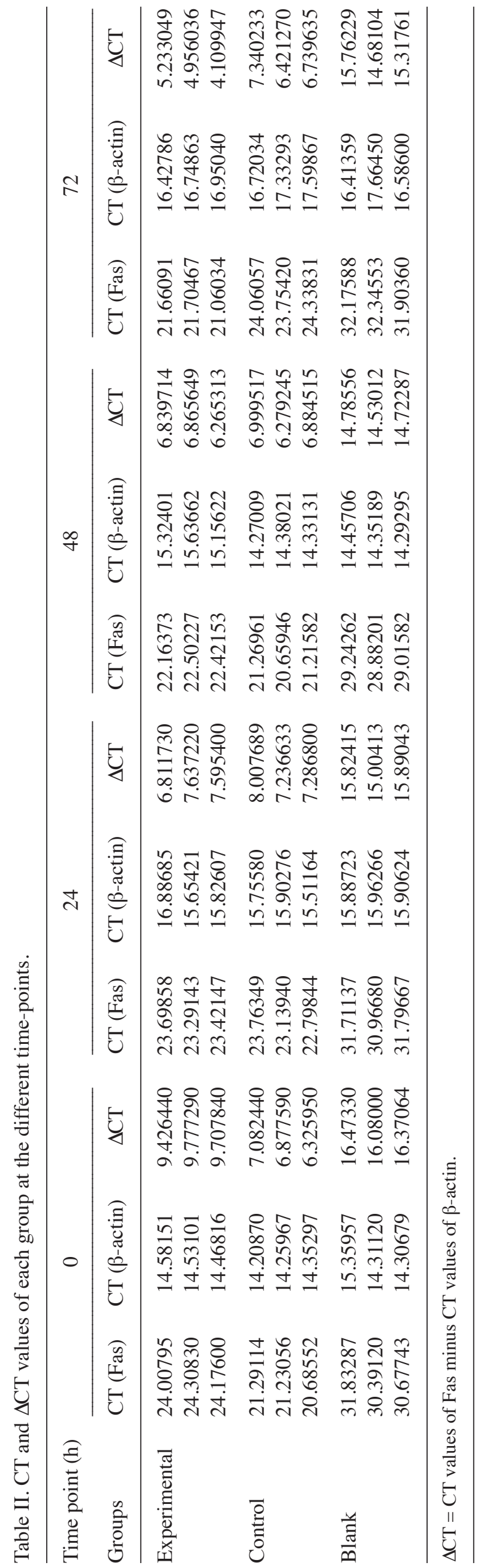

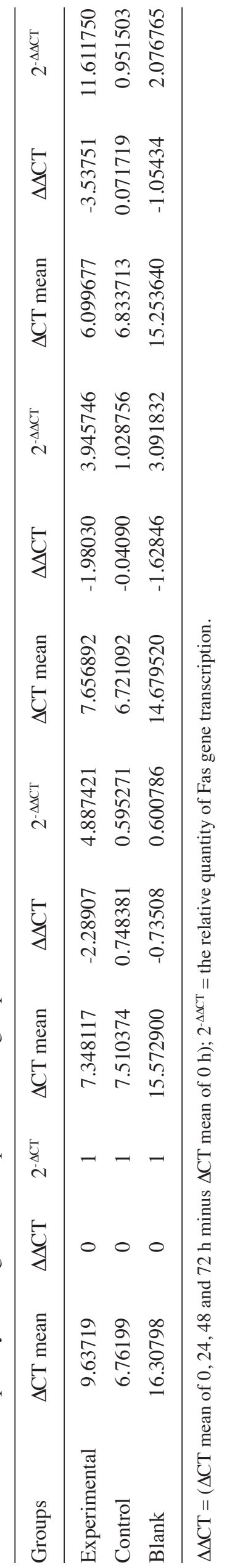


A

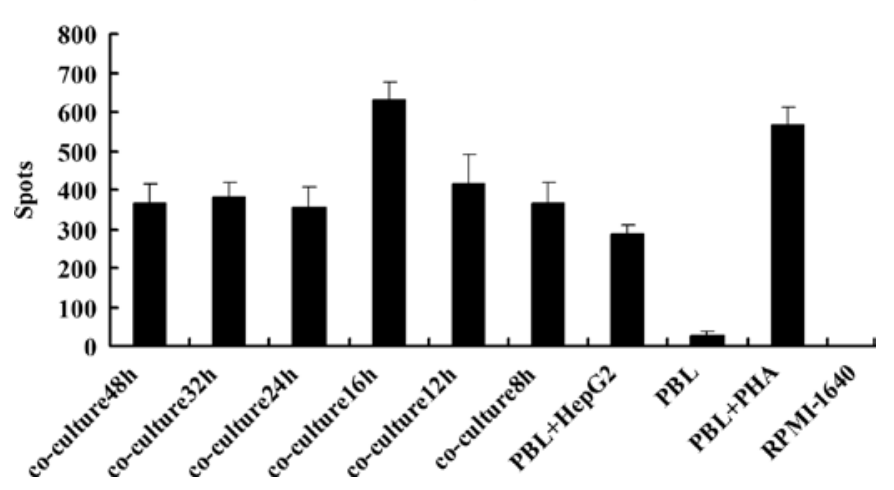

B

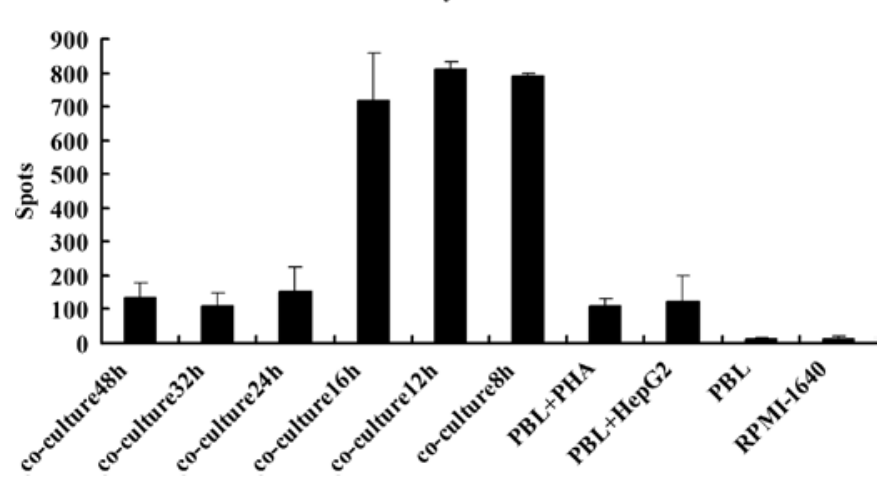

Figure 7. ELISPOT detection of TNF- $\alpha$ and IFN- $\gamma /$ IL2 at different times after pGPC3+afp-EGFP/HepG2 co-cultivation with PBLs.

at $16 \mathrm{~h}$, whereas that of IFN- $\gamma /$ IL2 appeared $12 \mathrm{~h}$ after the pGPC3+afp+EGFP/HepG2 cells co-cultivation with PBLs. The quantities of spots in some transfection groups were near to, or over, those in the positive controls (PHA stimulation groups) (Fig. 7).

\section{Disscusion}

AFP is often derepressed in human HCC, and it is an important serum marker in the diagnosis of HCC. The normal function of AFP is unknown, but it has been hypothesized to play a role in serum component transport as it has been shown to bind fatty acids, steroids, and heavy metals (7). The idea that AFP can serve as a target for immunotherapy is not novel. Efforts have been reported in earlier tumor immunology literature that involved attempts to generate humoral responses, but they were not successful due to high circulating levels of AFP neutralized antibody (3). Butterfield et al (2) reported that peptide fragments of AFP presented in the context of major histocompatibility molecules could serve as potential recognition targets by human $\mathrm{T}$ cell repertoire and characterized the response to an HLA-A2.1-restricted epitope hAFP $_{542-550}$ (GVALQTMKQ). Moreover, hAFP ${ }_{542-550}$ peptide-generated $\mathrm{T}$ cells from both human lymphocyte cultures and $\mathrm{A} 2.1 / \mathrm{Kb}$ transgenic mice recognized AFP-transfected targets in both cytotoxicity assays and cytokine release assays $(3,8)$. These findings clearly establish hAFP ${ }_{542-550}$ as a potential target for T cell-based immunotherapy.

Hepatoma cells, like other tumors, have a low immunogenicity and escape from human immunosurveillance and immune defence. Strong epitope $\mathrm{hAFP}_{542-550}$ expression on the exocy toplasmic membrane may help to improve the immunogenicity of hepatoma cells. GPC3 has been considered to be a new serum marker for the diagnosis of $\operatorname{HCC}(4,9,10)$, and its $\mathrm{C}$-terminus anchors to the exocytoplasmic surface of the cell membrane while the $\mathrm{N}$-terminus covering from 1 to 24 amino acids has secretion signal peptides according to the information in the SWISS-PROT database (No: P51654). Based on the 'protein engineering' theory, a recombinant pGPC 3+afp-EGFP was constructed and expressed successfully in our previous study (5). In that study, localization of pGPC3+afp-EGFP was further confirmed by fluorescence and confocal microscopy, Western blot analysis, and then its antitumor functions were thoroughly investigated.

When transfected HepG2 cells with pGPC3+afp-EGFP were co-cultured with PBLs, they became a kind of immunogenic particles which could strongly activate PBLs compared to the control groups. The proliferation of HepG2 cells in the experimental groups was significantly lower than those of the control and blank groups after $72 \mathrm{~h}$, as shown by SRB assay. The cell proliferative rate of the experimental group decreased with time according to the proliferation curve.

The Fas/FasL pathway is an important and intrinsic channel of apoptotic signal transduction. The Fas receptor is the key apoptotic regulator in antitumor immune effect. Substantially, the mRNA expression of apoptotic factors, such as caspase 3 had no change, whereas that of the death receptor, Fas, changed remarkably in the apoptotic assay and was consistent with apoptotic incidence $(11,12)$. Therefore, the mRNA transcription of the Fas gene was examined through fluorescence quantitative PCR at different time-points, and an increasing mRNA expression of the Fas gene in the experimental group was found. The mRNA overexpression of the Fas gene in the experimental group correlated with a significantly higher apoptotic incidence compared to the other two groups after $72 \mathrm{~h}$, as shown by statistical analysis and TUNEL assay.

IFN $\gamma$, IL- 2 and TNF- $\alpha$ are important antitumor cytokines related to humoral immune reaction. In the ELISPOT assays, IFN $\gamma /$ IL- 2 and TNF- $\alpha$ were induced by the transfected HepG2 cells with pGPC3+afp-EGFP (automated ELISPOT analyzer only read the spots of IL-2). Furthermore the optimum time was at 12-16 $\mathrm{h}$ after co-cultivation and the numbers of spots were more than those of the PHA stimulation groups.

Certain researchers had shown that GPC 3 could be a kind of T lymphocyte immune attack target in itself (13). Nakano et al (14) produced anti-GPC3 monoclonal antibody which could identify the C-terminal of GPC3, cause antibody-dependent cellular cytotoxicity (ADCC) and complement dependent cytotoxicity (CDC). Thus, GPC3 expression on the $\mathrm{HepG} 2$ cell membrane may induce antitumor immune response to some extent by itself, but based on the above results, we found that GPC3+afp-EGFP expression in the transfected HepG2 cells, whose strong epitope $\mathrm{hAFP}_{542-550}$ molecules were anchored in 
the cytoplasmic membrane, could produce overlapping antitumor effects.

However, more detailed antitumor evidence is required, such as both in vitro and in vivo. Studies on the further developments of GPC3 in other vectors with tumor-specific promoters, such as the AFPpromoter (15) or the telomerase promoter (16-17) are underway.

\section{Acknowledgements}

This study was supported by grants from the National Science Foundation of China (No. 30500239 and No. 81072037), and carried out partly in the National Laboratory of Medical Genetics of China. The authors sincerely thank academician Xia Jia-hui and everyone in the laboratory for their kind assistance.

\section{References}

1. Johnson PJ: The role of serum alpha-fetoprotein estimation in the diagnosis and management of hepatocellular carcinoma. Clin Liver Dis 5: 145-159, 2001.

2. Butterfield LH, Meng WS, Koh A, Vollmer CM, Ribas A Dissette V, Faull K, Glaspy JA, McBride WH and Economou JS: T cell responses to HLA-A*0201-restricted peptides derived from human alpha fetoprotein. J Immunol 8: 5300-5308, 2001.

3. Butterfield LH, Koh A, Meng W, Vollmer CM, Ribas A, Dissette V, Lee E, Glaspy JA, McBride WH and Economou JS: Generation of human T-cell responses to an HLA-A2.1-restricted peptide epitope derived from alpha-fetoprotein. Cancer Res 13: 3134-3142, 1999.

4. Capurro M, Wanless IR, Sherman M, Deboer G, Shi W, Miyoshi E and Filmus J: Glypican-3: a novel serum and histochemical marker for hepatocellular carcinoma. Gastroenterology 1: 89-97, 2003.

5. Yang DY, Li CH, Lan F, Zhang YQ, Lu FG, Yang ZR and Huang LQ: Expression and localization of strong epitope hAFP542-550 on eukaryotic cytoplasmic membrane. Xi Bao Yu Fen Zi Mian Yi Xue Za Zhi (in Chinese) 7: 592-595, 599, 2009.

6. Luo M, Zhou Y, Gong WD, Zhou SL, Zhang J and Zhang JR: Differences in chemosensitivity to anticancer drugs among hepatocarcinoma cells, bone marrow mesenchymal stem cells and HepG2 cells by SRB assay in rats. J Fourth Mil Med Univ 12: 1069-1070, 2007.
7. Mizejewski GJ: Physiology of alpha-fetoprotein as a biomarker for perinatal distress: relevance to adverse pregnancy outcome. Exp Biol Med 8: 993-1004, 2007.

8. Meng WS, Butterfield LH, Ribas A, Dissette VB, Heller JB, Miranda GA, Glaspy JA, McBride WH and Economou JS: alpha-Fetoprotein-specific tumor immunity induced by plasmid prime-adenovirus boost genetic vaccination. Cancer Res 24: 8782-8786, 2001.

9. Nakatsura T, Yoshitake Y, Senju S, Monji M, Komori H, Motomura Y, Hosaka S, Beppu T, Ishiko T, Kamohara H, et al: Glypican-3, overexpressed specifically in human hepatocellular carcinoma, is a novel tumor marker. Biochem Biophys Res Commun 1: 16-25, 2003.

10. Wang HL, Anatelli F, Zhai QJ, Adley B, Chuang ST and Yang XJ: Glypican-3 as a useful diagnostic marker that distinguishes hepatocellular carcinoma from benign hepatocellular mass lesions. Arch Pathol Lab Med 11: 1723-1728, 2008.

11. Mertlíková-Kaiserová H, Votruba I, Matousová M, Holy A and Hájek M: Role of caspases and CD95/Fas in the apoptotic effects of a nucleotide analog PMEG in CCRF-CEM cells. Anticancer Res 7: 2791-2798, 2010.

12. Yu J, Shen B, Chu ES, Teoh N, Cheung KF, Wu CW, Wang S, Lam CN, Feng $\mathrm{H}$, Zhao J, et al: Inhibitory role of peroxisome proliferator-activated receptor gamma in hepatocarcinogenesis in mice and in vitro. Hepatology 6: 2008-2019, 2010.

13. Komori H, Nakatsura T, Senju S, Yoshitake Y, Motomura Y, Ikuta Y, Fukuma D, Yokomine K, Harao M, Beppu T, et al: Identification of HLA-A2- or HLA-A24-restricted CTL epitopes possibly useful for glypican-3-specific immunotherapy of hepatocellular carcinoma. Clin Cancer Res 9: 2689-2697, 2006.

14. Nakano K, Orita T, Nezu J, Yoshino T, Ohizumi I, Sugimoto M, Furugaki K, Kinoshita Y, Ishiguro T, Hamakubo T, et al: Anti-glypiacan 3 antibodies cause ADCC Against human hepatocellular carcinoma cells. Biochem Biophys Res Commun 2: 279-284, 2009.

15. Cai X, Zhou J, Chang Y, Sun X, Li P and Lin J: Targeting gene therapy for hepatocarcinoma cells with the E. coli purine nucleoside phosphorylase suicide gene system directed by a chimeric alpha-fetoprotein promoter. Cancer Lett 1: 71-82, 2008.

16. Song MS, Jeong JS, Ban G, Lee JH, Won YS, Cho KS, Kim IH and Lee SW: Validation of tissue-specific promoter-driven tumor-targeting trans-splicing ribozyme system as a multifunctional cancer gene therapy device in vivo. Cancer Gene Ther 2: 113-125, 2009.

17. Zhang P, Chen Y, Jiang X, Tu Z and Zou L: Tumor-targeted efficiency of shRNA vector harboring chimera hTERT/U6 promoter. Oncol Rep 5: 1309-1316, 2010. 